\title{
ON THE SAINT VENANT PROBLEM FOR A NONHOMOGENEOUS ELASTIC MATERIAL*
}

\author{
BY \\ R. D. SCHILE AND R. L. SIERAKOWSKI \\ United Aircraft Corporation, East Hartford, Connecticut
}

Summary. The problem considered is the deformation of a cylindrical or prismatical bar, loaded by forces and moments applied at the ends, in which the elastic "constants" are arbitrary functions of two variables. The solution is formulated in terms of the six Beltrami stress functions and it is shown that two of these are sufficient to satisfy both compatibility and the boundary conditions.

When the variation of the elastic functions is uniformly small, and iterative method may be applied which yields the homogeneous elastic solution as a first approximation.

1. Introduction. In recent years there have appeared in the literature a small but steadily increasing number of papers concerned with the elasticity and plasticity of nonhomogeneous materials. Most of the problems considered have involved certain similifying assumptions of which may be mentioned the following:

a. One of the elastic functions, usually $\nu$ (Poisson's ratio) is assumed to be constant.

b. A particular type of inhomogeneity is assumed in order to simplify the equations.

c. Assumption of plane stress, plane strain or symmetry is made.

d. Assumptions regarding the vanishing of certain stresses are made and the elastic functions required to ensure compatibility are then determined.

For problems in which the boundary conditions are given in terms of the stresses, a general three-dimensional stress function theory has not been formulated.

The initial phases of the attack on the Saint Venant problem have already appeared in the literature. The torsion problem [1] has been formulated in terms of a single stress function assuming that both the shear modulus and Poisson's ratio are arbitrary functions of two variables. The shear modulus, however, is the only function influencing the determination of the stresses. The problem of bending of a prismatical bar [2] has been considered but restricted to the case $\nu=$ constant. The stresses are again given in terms of a single stress function and the governing differential equation is of the same form as for the torsion function. For variable $\nu$ it is apparent that the usual assumption that the transverse normal stresses vanish is invalid and the complete solution of this problem must therefore be based on a completely three-dimensional analysis.

The usual approach to three-dimensional problems in ordinary elasticity in which the surface tractions are specified is to write the stresses in terms of either the three Maxwell or the three Morera stress functions. The success of this method depends on the fact that the six compatibility equations, when written in terms of the stress functions, are reducible to three equations, subject only to the omission of certain additive functions [3], which do not affect the stresses. For a nonhomogeneous material, this reduction does not appear to be possible and one is forced to use the six Beltrami (Maxwell-Morera) functions.

*Received April 20, 1964. 
2. Three-dimensional stress functions. The compatibility equations in terms of the stresses for an isotropic, nonhomogeneous body are

$$
\begin{gathered}
{\left[\frac{\partial^{2}}{\partial x^{2}}+\frac{\partial^{2}}{\partial y^{2}}\right]\left[\frac{g \theta}{1+\nu}\right]-g \nabla^{2} \sigma_{z}-\frac{\partial^{2} g}{\partial x^{2}}\left(\sigma_{x}+\sigma_{z}\right)-\frac{\partial^{2} g}{\partial y^{2}}\left(\sigma_{y}+\sigma_{z}\right)} \\
-2 \frac{\partial^{2} g}{\partial x \partial y} \tau_{x y}+2 \frac{\partial g}{\partial x}\left(\frac{\partial \tau_{x z}}{\partial z}-\frac{\partial \sigma_{z}}{\partial x}\right)+2 \frac{\partial g}{\partial y}\left(\frac{\partial \tau_{y z}}{\partial z}-\frac{\partial \sigma_{z}}{\partial y}\right)=0, \\
\frac{\partial^{2}}{\partial y \partial z}\left[\frac{g \theta}{1+\nu}\right]+g \nabla^{2} \tau_{y z}+\frac{\partial^{2} g}{\partial x^{2}} \tau_{y z}-\frac{\partial^{2} g}{\partial x \partial y} \tau_{x z}-\frac{\partial^{2} g}{\partial x \partial z} \tau_{x y} \\
-\frac{\partial^{2} g}{\partial y \partial z}\left(\sigma_{\nu}+\sigma_{z}\right)+\frac{\partial g}{\partial x}\left(2 \frac{\partial \tau_{y z}}{\partial x}-\frac{\partial \tau_{x z}}{\partial y}-\frac{\partial \tau_{x y}}{\partial z}\right) \\
+\frac{\partial g}{\partial y}\left(\frac{\partial \tau_{y z}}{\partial y}-\frac{\partial \sigma_{y}}{\partial z}\right)+\frac{\partial g}{\partial z}\left(\frac{\partial \tau_{y z}}{\partial z}-\frac{\partial \sigma_{z}}{\partial y}\right)=0,
\end{gathered}
$$

where $g(x, y, z)=[G(x, y, z)]^{-1}, \nu=\nu(x, y, z), \theta=\sigma_{x}+\sigma_{\nu}+\sigma_{z}$. Two more equations of the form (1a) and two of the form (1b) are obtained by cyclic permutation of the variables $x, y, z$. By adding together all three equations of the type (1a), we obtain

$$
\begin{aligned}
\nabla^{2}\left[\left(\frac{1-\nu}{1+\nu}\right) g \theta\right]=\frac{\partial^{2}}{\partial x^{2}}\left(g \sigma_{x}\right) & +\frac{\partial^{2}}{\partial y^{2}}\left(g \sigma_{y}\right)+\frac{\partial^{2}}{\partial z^{2}}\left(g \sigma_{z}\right) \\
& +2\left[\frac{\partial^{2}}{\partial x \partial y}\left(g \tau_{x y}\right)+\frac{\partial^{2}}{\partial x \partial z}\left(g \tau_{x z}\right)+\frac{\partial^{2}}{\partial y \partial z}\left(g \tau_{y z}\right)\right] .
\end{aligned}
$$

The equilibrium equations are identically satisfied if the stresses are written in terms of the six Beltrami functions:

$$
\begin{array}{ll}
\sigma_{x}=B_{z z}+C_{y y}-2 L_{y z}, & \tau_{x y}=-C_{x y}+\left(L_{x}+M_{y}-N_{z}\right)_{z}, \\
\sigma_{y}=C_{x x}+A_{z z}-2 M_{x z}, & \tau_{x z}=-B_{x z}+\left(N_{z}+L_{x}-M_{y}\right)_{y}, \\
\sigma_{z}=A_{y y}+B_{x x}-2 N_{x y}, & \tau_{y z}=-A_{y z}+\left(M_{y}+N_{z}-L_{x}\right)_{x}
\end{array}
$$

The subscripts on $A, B, C, L, M, N$ denote partial derivatives. Substitution of Eq. (3) into the compatibility equations yields six equations in the six unknown stress functions. The resulting equations will not be written out as they are very lengthy. It is clear, however, that the presence of the terms involving derivatives of $g$ precludes the possibility of reducing this set of equations to a set of three equations in three unknowns, as is done in the classical case.

One may logically attempt such a reduction in the case $g=$ constant, $\nu=\nu(x, y, z)$. Taking $L \equiv M \equiv N \equiv 0$, we find

$$
\nabla^{2} A=\nabla^{2} B=\nabla^{2} C=\frac{\theta}{1+\nu} .
$$

For the problem at hand, it will be more convenient to take the compatibility equations in the form

$$
\begin{gathered}
\frac{\partial^{2}}{\partial x^{2}}\left(g \sigma_{y}\right)+\frac{\partial^{2}}{\partial y^{2}}\left(g \sigma_{x}\right)-\left(\frac{\partial^{2}}{\partial x^{2}}+\frac{\partial^{2}}{\partial y^{2}}\right)\left(\frac{g \nu}{1+\nu} \theta\right)=2 \frac{\partial^{2}}{\partial x \partial y}\left(g \tau_{x y}\right), \\
\frac{\partial^{2}}{\partial y \partial z}\left(g \sigma_{x}\right)-\frac{\partial^{2}}{\partial y \partial z}\left(\frac{g \nu}{1+\nu} \theta\right)=-\frac{\partial^{2}}{\partial x^{2}}\left(g \tau_{y z}\right)+\frac{\partial^{2}}{\partial x \partial y}\left(g \tau_{x z}\right)+\frac{\partial^{2}}{\partial x \partial z}\left(g \tau_{x y}\right)
\end{gathered}
$$


Two additional equations of the form (5a) and two of the form (5b) are obtained by cyclic permutation of the variables $x, y, z$.

3. The Saint Venant problem. Consider the problem of a cylindrical bar, loaded by forces and moments applied at the ends, in which $G=G(y, z), \nu=\nu(y, z)$, the variables $y, z$ being the coordinates in the plane of the cross section. The cylindrical boundary is to be free of tractions. We shall tentatively assume that the stress functions of Eq. (3) may be expanded into power series in $x$ and we shall retain only the first two terms.

$$
\begin{aligned}
A & =A^{0}(y, z)+x A^{\prime}(y, z), \\
\cdots & \ldots \ldots \ldots \cdots \cdots \cdots \cdots \cdots \\
N & =N^{0}(y, z)+x N^{\prime}(y, z), \\
\theta & =\theta^{0}(y, z)+x \theta^{\prime}(y, z), \\
\sigma_{x} & =\sigma_{x}^{0}(y, z)+x \sigma_{x}^{\prime}(y, z) .
\end{aligned}
$$

We substitute Eqs. (3) and (6) into Eqs. (5a) and (5b) and equate the coefficients of like powers of $x$.

Consider first the terms linear in $x$. The first, third and fourth of the compatibility equations yield

$$
\sigma_{x}^{\prime}=E\left(\beta_{1}+\beta_{2} y+\beta_{3} z\right)+\nu \nabla_{1}^{2} A^{\prime},
$$

where the $\beta$ 's are constants, $E$ is Young's modulus and $\nabla_{1}^{2} \equiv \partial^{2} / \partial y^{2}+\partial^{2} / \partial z^{2}$. From the second compatibility equation we have, making use of Eq. (7),

$\frac{\partial^{2}}{\partial y^{2}}\left(g A_{y y}^{\prime}\right)+\frac{\partial^{2}}{\partial z^{2}}\left(g A_{z z}^{\prime}\right)+2 \frac{\partial^{2}}{\partial y \partial z}\left(g A_{\nu z}^{\prime}\right)-\nabla_{1}^{2}\left(g \nu \nabla_{1}^{2} A^{\prime}\right)=\nabla_{1}^{2}\left[2 \nu\left(\beta_{1}+\beta_{2} y+\beta_{3} z\right)\right]$

Finally, from the last two compatibility equations, we find

$$
\frac{\partial}{\partial y}\left(g \phi_{y}^{\prime}\right)+\frac{\partial}{\partial z}\left(g \phi_{z}^{\prime}\right)=\gamma,
$$

where $\phi^{\prime}=N_{z}^{\prime}-M_{y}^{\prime}$, and $\gamma$ is a constant.

A similar accounting of the terms independent of $x$ produces the following equations:

$$
\sigma_{x}^{0}=E\left[\alpha_{1}+\alpha_{2} y+\alpha_{3} z+\frac{\gamma}{2} y z-\int g \phi_{z}^{\prime} d y\right]+\nu \nabla_{1}^{2} A^{0}-2 \nu\left(M_{z}^{\prime}+N_{y}^{\prime}\right),
$$

where $\alpha_{1}, \alpha_{2}, \alpha_{3}$ are constants,

$$
\begin{aligned}
& \frac{\partial^{2}}{\partial y^{2}}\left(g A_{y y}^{0}\right)+\frac{\partial^{2}}{\partial z^{2}}\left(g A_{z z}^{0}\right)+2 \frac{\partial^{2}}{\partial y \partial z}\left(g A_{y z}^{0}\right)-\nabla_{1}^{2}\left(g \nu \nabla_{1}^{2} A^{0}\right) \\
& \quad=\nabla_{1}^{2}\left[2 \nu\left(\alpha_{1}+\alpha_{2} y+\alpha_{3} z+\frac{\gamma}{2} y z-\int g \phi_{z}^{\prime} d y\right)\right] \\
& \quad+2 \frac{\partial^{2}}{\partial y^{2}}\left(g N_{y}^{\prime}\right)+2 \frac{\partial^{2}}{\partial z^{2}}\left(g M_{z}^{\prime}\right)+2 \frac{\partial^{2}}{\partial y \partial z}\left[g\left(M_{\nu}^{\prime}+N_{z}^{\prime}\right)\right]-2 \nabla_{1}^{2}\left[g \nu\left(M_{z}^{\prime}+N_{y}^{\prime}\right)\right] \\
& \frac{\partial}{\partial y}\left[\frac{\partial}{\partial y}\left(g \phi_{y}^{0}\right)+\frac{\partial}{\partial z}\left(g \phi_{z}^{0}\right)\right]=\frac{\partial}{\partial z}\left[2 \nu\left(\beta_{1}+\beta_{2} y+\beta_{3} z\right)\right] \\
& -\frac{\partial}{\partial z}\left[g\left(A_{z z}^{\prime}-\nu \nabla_{1}^{2} A^{\prime}\right)\right]-\frac{\partial}{\partial y}\left(g A_{y_{z}}^{\prime}\right)-\frac{\partial}{\partial y}\left[\frac{\partial}{\partial y}\left(g\left(L_{y}^{\prime}-B_{z}^{\prime}\right)\right)-\frac{\partial}{\partial z}\left(g\left(L_{z}^{\prime}-C_{\nu}^{\prime}\right)\right)\right]
\end{aligned}
$$


In view of the fact that $\sigma_{x}$ is found directly, there is no need for all three stress functions $B, C, L$ and we may take $B \equiv L \equiv 0$, deleting these terms from Eq. (12).

The boundary conditions must be such that the cylindrical boundary is free of tractions. In addition, at any section $x=$ constant, the resultant axial load, two bending moments, twisting moment and two shear forces are specified. Since the solution contains 6 contants $\alpha_{1} \cdots \beta_{3}$ it would seem that the constant $\gamma$ appearing in Eq. (9) is not needed and we shall assume $\gamma=0$. The solution may then be further specialized by taking $M^{\prime} \equiv N^{\prime} \equiv \phi^{\prime} \equiv 0$. Equations (7) through (12) then appear in the simplified form

$$
\begin{gathered}
\sigma_{x}=E\left[\left(\alpha_{1}+\beta_{1} x\right)+\left(\alpha_{2}+\beta_{2} x\right) y+\left(\alpha_{3}+\beta_{3} x\right) z\right]+\nu \nabla_{1}^{2} A, \\
\begin{aligned}
& \frac{\partial^{2}}{\partial y^{2}}\left(g A_{y y}\right)+\frac{\partial^{2}}{\partial z^{2}}\left(g A_{z z}\right)+ 2 \frac{\partial^{2}}{\partial y \partial z}\left(g A_{y z}\right)-\nabla_{1}^{2}\left(g \nu \nabla_{1}^{2} A\right) \\
&=\nabla_{1}^{2}\left[2 \nu\left(\left(\alpha_{1}+\beta_{1} x\right)+\left(\alpha_{2}+\beta_{2} x\right) y+\left(\alpha_{3}+\beta_{3} x\right) z\right)\right], \\
& \frac{\partial}{\partial y}\left[\frac{\partial}{\partial y}\left(g \phi_{y}^{0}\right)+\frac{\partial}{\partial z}\left(g \phi_{z}^{0}\right)\right]= \frac{\partial}{\partial z}\left[2 \nu\left(\beta_{1}+\beta_{2} y+\beta_{3} z\right)\right] \\
&-\frac{\partial}{\partial z}\left[g\left(A_{z z}^{\prime}-\nu \nabla_{1}^{2} A^{\prime}\right)\right]-\frac{\partial}{\partial y}\left(g A_{y z}^{\prime}\right)-\frac{\partial^{2}}{\partial y \partial z}\left[g \int \sigma_{x}^{\prime} d y\right]
\end{aligned}
\end{gathered}
$$

Note that the separate equations for $\sigma_{x}^{0}, \sigma_{x}^{1}$ and $A^{0}, A^{\prime}$ have been incorporated into single equations. The remaining stresses are determined from the relations

$$
\begin{gathered}
\sigma_{y}=A_{z z}, \quad \sigma_{z}=A_{y y} \\
\tau_{x y}=-\int \sigma_{x}^{\prime} d y-\phi_{z}^{0}, \quad \tau_{x z}=\phi_{y}^{0}, \quad \tau_{y z}=-A_{y z},
\end{gathered}
$$

It will be of interest to examine several special cases of this solution.

(a) The case $\nu=$ constant. The right-hand side of Eq. (13b) vanishes and we may then take $A^{0} \equiv A^{\prime} \equiv 0$. The remaining equation for the stress function $\phi^{0}$ may then be integrated with respect to $y$.

$$
\frac{\partial}{\partial y}\left(g \phi_{y}^{0}\right)+\frac{\partial}{\partial z}\left(g \phi_{z}^{0}\right)=2 \nu \beta_{3} y+f(z)-\frac{\partial}{\partial z}\left[g \int E\left(\beta_{1}+\beta_{2} y+\beta_{3} z\right) d y\right],
$$

where $f(z)$ is an arbitrary function. The stresses are completely determined by this equation, the solution being

$$
\begin{aligned}
\sigma_{x} & =E\left[\left(\alpha_{1}+\beta_{1} x\right)+\left(\alpha_{2}+\beta_{2} x\right) y+\left(\alpha_{3}+\beta_{3} x\right) z\right], \\
\sigma_{y} & \equiv \sigma_{z} \equiv \tau_{y z} \equiv 0, \\
\tau_{x y} & =-\left(\beta_{1}+\beta_{3} z\right) \int E d y-\beta_{2} \int y E d y-\phi_{z}^{0}, \\
\tau_{x z} & =\phi_{y}^{0} .
\end{aligned}
$$

This solution coincides esssentially with that given by Schile [2].

(b) Pure torsion. Let us suppose that $A \equiv 0$ and $\alpha_{1}=\cdots=\beta_{3}=0$ then Eqs. (13a) and $(13 \mathrm{~b})$ are satisfied and $(13 \mathrm{c})$ becomes

$$
\frac{\partial}{\partial y}\left(g \phi_{\sharp !}^{0}\right)+\frac{\partial}{\partial z}\left(g \phi_{z}^{0}\right)=f(z)
$$


The stresses are

$$
\tau_{x y}=-\phi_{z}^{0}, \quad \tau_{x z}=\phi_{y}^{0}, \quad \sigma_{x} \equiv \sigma_{y} \equiv \sigma_{z} \equiv \tau_{y z} \equiv 0 .
$$

(c) Simple tension. If we take $\beta_{1}=\beta_{2}=\beta_{3}=0$ in Eqs. (13a)-(13c), we obtain

$$
\begin{gathered}
\sigma_{x}^{0}=E\left[\alpha_{1}+\alpha_{2} y+\alpha_{3} z\right]+\nu \nabla_{1}^{2} A^{0}, \quad \sigma_{x}^{\prime} \equiv 0, \quad A^{\prime} \equiv 0, \\
\frac{\partial^{2}}{\partial y^{2}}\left(g A_{y y}^{0}\right)+\frac{\partial^{2}}{\partial z^{2}}\left(g A_{z z}^{0}\right)+2 \frac{\partial^{2}}{\partial y \partial z}\left(g A_{y z}^{0}\right)-\nabla_{1}^{2}\left(g \nu \nabla_{1}^{2} A^{0}\right) \\
=\nabla_{1}^{2}\left[2 \nu\left(\alpha_{1}+\alpha_{2} y+\alpha_{3} z\right)\right], \\
\frac{\partial}{\partial y}\left(g \phi_{y}^{0}\right)+\frac{\partial}{\partial z}\left(g \phi_{z}^{0}\right)=f(z) .
\end{gathered}
$$

If $f(z) \equiv 0$, then $\phi^{0} \equiv 0$ and the shearing stresses $\tau_{x y}, \tau_{x z}$ are both zero. It can be seen that we require a non-trivial solution for $A^{0}$ and therefore the stresses $\sigma_{y}, \sigma_{z}, \tau_{y z}$ do not vanish. These stresses exist because of the lateral constraint introduced by the varying $\nu$ and they vanish when $\nu$ is a constant.

(d) Pure bending. This case is exactly the same as for simple tension. The three constants $\alpha_{1}, \alpha_{2}, \alpha_{3}$ are determined from the relations

$$
\int_{S} \sigma_{x}^{0} d S=0, \quad \int_{S} y \sigma_{x}^{0} d S=M, \quad \int_{S} z \sigma_{x}^{0} d S=0,
$$

while for simple tension, these conditions are given in the form

$$
\int_{S} \sigma_{x}^{0} d S=T, \quad \int_{S} y \sigma_{x}^{0} d S=0, \quad \int_{S} z \sigma_{x}^{0} d S=0 .
$$

The same remarks concerning the lateral constraint stresses apply here also.

It should be pointed out that in pure bending, $\alpha_{1}$ is not necessarily zero and in simple tension, $\alpha_{2}, \alpha_{3}$ are not necessarily zero so that tension and pure bending are, in general, coupled. Similarly, bending by a transverse load is coupled with both tension and torsion.

(e) Plane stress-Bar of narrow section. If we require $\sigma_{z}, \tau_{x z}, \tau_{y z}$ to vanish, $A$ and $\phi^{0}$ must be identically zero. The constant $\beta_{3}$ is not necessary and we may set this equal to zero also. Equations (13a)-(13d) are then reduced to

$$
\begin{aligned}
& \sigma_{x}=E\left[\left(\alpha_{1}+\beta_{1} x\right)+\left(\alpha_{2}+\beta_{2} x\right) y\right], \\
& \sigma_{y} \equiv 0, \\
& \tau_{x y}=-\int E\left(\beta_{1}+\beta_{2} y\right) d y+\text { const, } \\
& \nabla_{1}^{2}\left[2 \nu\left(\left(\alpha_{1}+\beta_{1} x\right)+\left(\alpha_{2}+\beta_{2} x\right) y\right)\right]=0,
\end{aligned}
$$

where $E=E(y), \nu=\nu(y)$.

The last of the above relations constitutes an incompatibility which is satisficd only when $\nu(y)$ is appropriately restricted. This, of course, agrees with the well-known fact that the plane stress state in the classical theory $(g, \nu$ constant) does not satisfy the compatibility equations exactly. If we ignore the equation involving $\nu$, the stresses given above agree exactly with the known solution for a thin bar with an end load [4]. 
The above incompatibility may be obtained in a more recognizable form if we consider a different interpretation of the solution given by Eqs. (13a)-(13d). Let $y, z$ be the coordinates in the plane of a thin plate and $x$ be the coordinate normal to the plate. As before, $g=g(y, z), \nu=\nu(y, z)$. We now attempt to specialize Eqs. (13) in order to obtain a state of plane stress, $\sigma_{x} \equiv \tau_{x y} \equiv \tau_{x z} \equiv 0$. Let all six constants $\alpha_{1}, \cdots \beta_{3}$ vanish. Also, let $A^{\prime} \equiv 0$. Then $\sigma_{x}^{\prime} \equiv 0$ and we may take a particular solution of Eq. (13c) as $\phi^{0} \equiv 0$. Remaining are

$$
\begin{gathered}
\sigma_{x}=\nu \nabla_{1}^{2} A^{0}, \quad \sigma_{y}=A_{z z}^{0}, \quad \sigma_{z}=A_{y y}^{0}, \quad \tau_{y z}=-A_{y z}^{0}, \\
\frac{\partial^{2}}{\partial y^{2}}\left(g A_{y y}^{0}\right)+\frac{\partial^{2}}{\partial z^{2}}\left(g A_{z z}^{0}\right)+2 \frac{\partial^{2}}{\partial y \partial z}\left(g A_{y z}^{0}\right)-\nabla_{1}^{2}\left(g \nu \nabla_{1}^{2} A^{0}\right)=0 .
\end{gathered}
$$

The normal stress $\sigma_{x}$ does not vanish unless $A^{0}$ is harmonic, which is the same incompatibility that arises in the classic case. The identical result may also be obtained from Eqs. (7) through (12) by the following substitutions:

$$
\begin{gathered}
\alpha_{1}=\cdots=\beta_{3}=0, \quad A^{0} \equiv A^{\prime} \equiv 0, \quad M^{0} \equiv N^{0} \equiv 0 \\
B \equiv C \equiv L \equiv 0, \quad \phi^{\prime}=N_{z}^{\prime}-M_{\nu}^{\prime}=0, \quad\left(-2 N^{\prime}=\psi_{y}^{\prime},-2 M^{\prime}=\psi_{z}^{\prime}\right) .
\end{gathered}
$$

The same relations are obtained except with $A^{0}$ replaced by $\psi^{\prime}$.

4. Solution of the equations. Equations (13b) and (13c) constitute a set of two partial differential equations with variable coefficients. If $g$ and $\nu$ are arbitrary functions, it is impossible to establish any general results concerning the character of the solutions.

When $g$ and $\nu$ are constants these equations are reduced respectively to the biharmonic equation and the membrane equation. Since the solutions of these equations are known, it is logical to attempt to reduce the nonhomogeneous case to a succession of homogeneous elasticity problems. The perturbation procedure used to accomplish this end is wellknown and has been used extensively to obtain approximate solutions of problems in other areas. We assume

$$
G=G_{0}(1+\epsilon \lambda(y, z)), \quad \nu=\nu_{0}(1+\epsilon \mu(y, z)),
$$

where $\lambda(y, z), \mu(y, z)$ are arbitrary functions; $G_{0}, \nu_{0}$ are constants and $\epsilon$ is a small parameter. Substituting Eq. (14) into Eqs. (13b) and (13c), we obtain

$$
\begin{aligned}
\left(1-\nu_{0}\right) \nabla_{1}^{4} A- & \epsilon\left[\frac{\partial^{2}}{\partial y^{2}}\left(\lambda A_{y y}\right)+\frac{\partial^{2}}{\partial z^{2}}\left(\lambda A_{z z}\right)+2 \frac{\partial^{2}}{\partial y \partial z}\left(\lambda A_{y z}\right)+\nu_{0} \nabla_{1}^{2}\left[(\lambda-\mu) \nabla_{1}^{2} A\right]\right] \\
+ & O\left(\epsilon^{2}\right)=2 \epsilon G_{0} \nu_{0} \nabla_{1}^{2}\left[\mu\left(\left(\alpha_{1}+\beta_{1} x\right)+\left(\alpha_{2}+\beta_{2} x\right) y+\left(\alpha_{3}+\beta_{3} x\right) z\right)\right] \\
\frac{\partial}{\partial y} \nabla_{1}^{2} \phi^{0}-\epsilon \frac{\partial}{\partial y} & {\left[\frac{\partial}{\partial y}\left(\lambda \phi_{y}^{0}\right)+\frac{\partial}{\partial z}\left(\lambda \phi_{z}^{0}\right)\right]=2 \nu_{0} G_{0} \beta_{3}-\left(1-\nu_{0}\right) \nabla_{1}^{2} A_{z}^{\prime}-\frac{\partial \sigma_{x}^{\prime}}{\partial z} } \\
+ & \left\{2 \nu_{0} G_{0} \frac{\partial}{\partial z}\left[\mu\left(\beta_{1}+\beta_{2} y+\beta_{3} z\right)\right]+\frac{\partial}{\partial z}\left[\lambda A_{z z}^{\prime}+\nu_{0}(\mu-\lambda) \nabla_{1}^{2} A^{\prime}\right]\right. \\
& \left.+\frac{\partial}{\partial y}\left(\lambda A_{y z}^{\prime}\right)+\frac{\partial^{2}}{\partial y \partial z}\left[\lambda \int \sigma_{x}^{\prime} d y\right]\right\}
\end{aligned}
$$

Since the stress function $A$ determines only the constraint stresses and since these stresses vanish when $\epsilon=0$, we may assume that $A=O(\epsilon)$. Then

$$
\begin{aligned}
& \phi^{0}=\psi^{0}+\epsilon \chi^{0}+O\left(\epsilon^{2}\right), \\
& A=A^{0}+A^{\prime} x=\epsilon\left(\Omega^{0}+\Omega^{\prime} x\right)+O\left(\epsilon^{2}\right) .
\end{aligned}
$$


Using Eqs. (15) and (16), we find

$$
\begin{gathered}
\frac{\partial}{\partial y} \nabla_{1}^{2} \psi^{0}=-\frac{\partial}{\partial z} \sigma_{x}^{\prime 0}+2 \nu_{0} G_{0} \beta_{3}, \\
\frac{\partial}{\partial y} \nabla_{1}^{2} \chi^{0}=\frac{\partial}{\partial y}\left[\frac{\partial}{\partial y}\left(\lambda \psi_{y}^{0}\right)+\frac{\partial}{\partial z}\left(\lambda \psi_{z}^{0}\right)\right]-\left(1-\nu_{0}\right) \nabla_{1}^{2} \Omega_{z}^{\prime} \\
+2 \nu_{0} G_{0} \frac{\partial}{\partial z}\left[\mu\left(\beta_{1}+\beta_{2} y+\beta_{3} z\right)\right]+\frac{\partial^{2}}{\partial y \partial z}\left[\lambda \int \sigma_{x}^{\prime \prime} d y\right], \\
\left(1-\nu_{0}\right) \nabla_{1}^{4} \Omega=2 G_{0} \nu_{0} \nabla_{1}^{2}\left[\mu\left(\left(\alpha_{1}+\beta_{1} x\right)+\left(\alpha_{2}+\beta_{2} x\right) y+\left(\alpha_{3}+\beta_{3} x\right) z\right)\right],
\end{gathered}
$$

where

$$
\begin{aligned}
& {\sigma_{x}^{\prime 0}}^{\prime 0}=E_{0}\left(\beta_{1}+\beta_{2} y+\beta_{3} z\right), \\
& \sigma_{x}^{\prime \prime}=\left(2 G_{0} \nu_{0}(\lambda+\mu)+2 G_{0} \lambda\right)\left(\beta_{1}+\beta_{2} y+\beta_{3} z\right)+\nu_{0} \nabla_{1}^{2} \Omega^{\prime}, \\
& E_{0}=2 G_{0}\left(1+\nu_{0}\right) .
\end{aligned}
$$

Terms involving higher powers of $\epsilon$ may be retained if desired, but the essential features of the solution are given by the initial terms. It is apparent that, in addition to the assumption that $\epsilon$ is small, we must also require that the quantities $\chi^{0}$ and $\Omega$ be everywhere small. The derivatives of $\lambda$ and $\mu$ must therefore not be too large throughout the considered region.

5. Concluding remarks. We have shown that the state of stress in a bar with planar inhomogeneity has a three-dimensional character, even in the state of simple tension. The constraint stresses exist because of the variable Poisson contraction and disappear when $\nu$ is a constant.

The use of the Beltrami functions offers a general approach to the solution of problems in three-dimensional nonhomogeneous elasticity. It would seem that, in the case $\nu=$ constant, a reduction in the number of stress functions from six to three should be possible, similar to that derived herein for the case $G=$ constant. This expectation has, however, not yet been proven.

\section{REFERENCES}

1. J. F. Ely and O. C. Zienkiewicz, Int'l. Jl. Mech. Sci. 1 (1960) 356

2. R. D. Schile, Int'l. Jl. Mech. Sci. 5 (1963) 439

3. H. L. Langhaar and M. Stippes, Jl. Franklin Inst. 258 (1954) 371

4. R. D. Schile, Jl. Appl. Mech. 29 (1962) 582 